\title{
Open Abdomen during Extracorporeal Membrane Oxygenation is a Safe and Effective Treatment for Abdominal Compartment Syndrome
}

\author{
Joshua Brown ${ }^{1}$; Brielle Warnock2; Eamaan Turk ${ }^{3}$; Gail Hocutt RNC4; Brian W Gray, MD ${ }^{5}$ \\ ${ }^{1}$ Indiana University School of Medicine, ${ }^{2}$ Indiana University School of Medicine, ${ }^{3}$ Dow Medical \\ College; ${ }^{4}$ Riley Hospital for Children, ECMO Program Manager, ${ }^{5}$ Indiana University School of \\ Medicine, Department of Surgery, Division of Pediatric Surgery
}

\section{Background/Purpose}

Historically, decompressive laparotomy and open abdomen for abdominal compartment syndrome has contraindicated Extracorporeal Membrane Oxygenation (ECMO) due to seemingly high risk of bleeding and infection. The literature shows few examples of this treatment, and the existing studies are inconclusive. The purpose of this study was to review the series at Riley Hospital for Children and evaluate the effectiveness of ECMO treatment for patients undergoing decompressive laparotomy with open abdomen to recommend future care guidelines.

\section{Methods}

We reviewed all pediatric ( 30 days to 18 years) patients treated with ECMO concurrently with decompressive laparotomy and open abdomen at Riley Hospital for Children from 2000-2019. We compared these patients with non-surgical pediatric patients supported with ECMO for respiratory failure at Riley Hospital for Children during the same period. Demographics, ECMO data, and outcomes were assessed. We performed t-test, ROC, and chi-square analyses. We defined significance as $p=0.05$.

\section{Results}

5 of 82 pediatric respiratory ECMO patients were treated with decompressive laparotomy and open abdomen. Survival among the surgical group was $60 \%$, compared to $57 \%$ in the nonsurgical group $(p=0.9)$. Surgical patients had a similar incidence of bleeding complications $(40 \%)$ compared to non-surgical patients $(55.8 \%), p=0.486$. Surgical patients had a significantly higher VIS (3126 vs 19.2, p=0.004), $\mathrm{PaO}_{2} / \mathrm{FiO}_{2}$ ratio (279.0 vs $72.9, \mathrm{p}=0.031$ ), and pump flow rate at $24 \mathrm{hrs}(112 \mathrm{~mL} / \mathrm{kg} / \mathrm{min}$ vs $88.1 \mathrm{~mL} / \mathrm{kg} / \mathrm{min}, p=0.045)$ than non-surgical patients, while receiving a similar volume of PRBCs $(p=0.581)$ and requiring $E C M O$ treatment for a similar amount of time $(p=0.511)$.

\section{Conclusion/Potential Impact}

ECMO support in patients with decompressive laparotomy and open abdomen was associated with similar survival and bleeding complications compared to non-surgical ECMO patients. ECMO should be offered to or continued in eligible patients with abdominal catastrophe, as it is effective in supporting organ function while not significantly increasing the risk for complications. 